increased CT and/or NG risk $(\mathrm{aHR}=1.03, \mathrm{p}<0.001)$, and men reporting having sex in exchange for money at screening were 2.35 times more likely to be infected $(p=0.072)$. Number of sex partners, sexual positioning, condom use, lubricant use, and self-reported PrEP adherence were not associated with infection.

Conclusion The incidence of urethral CT/NG infection was high in our cohort of MSM taking PrEP, despite risk reduction counselling and repeated testing and treatment, supporting need for ongoing etiologic testing and more effective risk reduction intervention. Additional analyses will examine risk compensation.

Disclosure No significant relationships.

\section{P454 EMERGENCY DEPARTMENT (ED)-BASED HIV PRE- EXPOSURE PROPHYLAXIS (PREP) REFERRAL PROGRAM - USING EDS AS A PORTAL FOR PREP SERVICES}

\begin{abstract}
'Zezhou Zhao*, ${ }^{2}$ Joyce Jones, ${ }^{3}$ Renata Sanders, 'Gaby Gladfelter, ${ }^{2}$ Steven Mcdonald, ${ }^{3}$ Christopher Reed, ${ }^{1}$ Jojo Castellanos, ${ }^{3}$ Glenn Fulton, ${ }^{1}$ Kaitlynn Motley, ${ }^{1}$ Eric Campbell, ${ }^{1}$ Deanna Myer, 'Tiana Jones, ${ }^{1}$ Richard Rothman, ${ }^{1} Y u$-Hsiang Hsieh. ${ }^{1} J o h n s$ Hopkins University School of Medicine, Department of Emergency Medicine, Baltimore, USA; ${ }^{2}$ Johns Hopkins University School of Medicine, Division of Infectious Diseases, Baltimore, USA; ${ }^{3}$ Johns Hopkins University School of Medicine, Division of General Pediatrics and Adolescent Medicine, Department of Pediatrics, Baltimore, USA
\end{abstract}

\subsection{6/sextrans-2019-sti.537}

Background U.S. Emergency Departments (EDs) serve many patients at high risk for HIV and could serve as a portal of entry for pre-exposure prophylaxis (PrEP). We conducted a pilot study to determine feasibility and acceptability of PrEP referral from the ED.

Methods From late December 2018 to March 2019, a pilot PrEP referral program was integrated into an established HIV/ HCV screening program in an urban U.S. ED with HIV seroprevalence of $6 \%$. Patients were deemed PrEP referral eligible if they had recent STI-related visit ( $<2$ months) and tested HIV negative, or a negative HIV test during the current ED visit. The PrEP referral program was implemented by HIV/ HCV program staff and trained volunteers. Patients were approached by convenience sampling based on staff availability. HIV risk and interest in PrEP referral was assessed through an electronic survey based on CDC PrEP guidelines. Patients expressing interest in PrEP were referred to PrEP peer navigators from the adult infectious disease specialty clinic and pediatric clinic. Referrals, scheduled and completed appointments, and PrEP initiation were collected.

Results In this ongoing program, 290 ED patients were screened, 162 approached and 89 (55\%) took the survey. 68 (42\%) were categorized as high-risk and eligible for PrEP referral. Of these 68 patients, 36 (53\%) accepted PrEP referral, 15 (22\%) were successfully contacted by phone with additional 21 (31\%) pending for follow-up calls, six (9\%) were scheduled for appointments, and two (3\%) completed an appointment.

Conclusion Our preliminary findings demonstrate a high interest for PrEP referral and comparable PrEP care cascade outcomes in high-risk ED patients indicating that implementation of an ED-based PrEP referral program is feasible and acceptable and could serve as an important portal for PrEP care. Barriers along the PrEP care continuum to better engage and retain patients should be addressed as a future direction.

Disclosure No significant relationships.

\section{P455 AWARENESS, KNOWLEDGE AND WILLINGNESS TO USE HIV PREP AMONG MSM AND TG PEOPLE IN BALI, INDONESIA}

Lucyan Umboh, Irvin Romyco*. FHI360-LINKAGES, COPTC (Continuum of HIV Prevention, Treatment, and (are), Jayapura, Indonesia

\subsection{6/sextrans-2019-sti.538}

Background World Health Organisation in 2014 has recommended Pre-Exposure Prophylaxis (PrEP) as HIV prevention tool for men who have sex with men (MSM) along with other prevention strategies. Before PrEP implementation is introduced in Indonesia, it is vitally important to have an evidence base about the awareness, knowledge and acceptability among MSM and transgender people and the differences in knowledge and PrEP acceptability based on sexual risk factors of MSM and transgender people.

Methods A cross-sectional study was conducted between July and November 2017 in an MSM clinic in Denpasar, Bali, Indonesia. The key outcomes were exploring the awareness, knowledge and willingness to use PrEP among MSM and transgender people in Bali, Indonesia and determining demographic and sexual risk factors associated with knowledge and willingness to use PrEP.

Results A total of 164 participants were recruited into the study but only 124 participants were eligible and included in the analysis. Of these, 69 out of 124 (55.6\%) participants had never heard about PrEP. Among those 55 (44.4\%) participants who had heard about PrEP, education and occupation were the only factors associated with PrEP knowledge. Men with the University education showed higher knowledge scores (29.2) and were more likely to use PrEP compared with men with lower education levels. 73 out of 124 participants (58.9\%) were willing to take PrEP in the future and education was the only factor associated with PrEP willingness.

Conclusion This is the first study to report data on awareness, knowledge and willingness to use PrEP in Bali and is only the second such study conducted in Indonesia. Consistent with similar studies in other countries, this study found limited knowledge of PrEP among MSM and transgender participants. As PrEP is not yet publicly available in Indonesia, it is crucially important to prepare the community by education and disseminating accurate information.

Disclosure No significant relationships.

\section{P457 A SINGLE DOSE OF BEHAVIOUR CHANGE: THE IMPACT OF TESTING ON BEHAVIOUR AND CHLAMYDIA TRANSMISSION}

${ }^{1}$ Daphne Van Wees*, ${ }^{2}$ Chantal Den Daas, 'Janneke Heijne, ${ }^{3}$ Mirjam Kretzschmar. 'National Institute for Public Health and the Environment (RIVM), Epidemiology and Surveillance, Centre for Infectious Diseases Control, Bilthoven, Netherlands; '2Utrecht University, Department of Interdisciplinary Social Science, Faculty of Social and Behavioural Sciences, Utrecht, Netherlands; ${ }^{3}$ University Medical Centre Utrecht, Julius Centre for Health Sciences and Primary Care, Utrecht, Netherlands

\subsection{6/sextrans-2019-sti.539}

Background The aim was to explore short-term effects of chlamydia testing on sexual behaviour also taking into account psychological characteristics, and to estimate the impact on chlamydia transmission of interventions aimed at sustaining or enhancing testing effects. 